\title{
The Empirical Analysis of Teaching Quality Guarantee System for Universities Based on the Fuzzy Comprehensive Evaluation and Fuzzy Neural Network Models
}

\author{
Chunfeng Liu', a ${ }^{1,}$ Peiluan $\mathrm{Li}^{2, \mathrm{~b}}$ and Baoan $\mathrm{Li}^{2, \mathrm{c}^{*}}$ \\ ${ }^{1}$ Academic Administration Department, Henan University of Science and Technology, Luoyang, \\ China \\ ${ }^{2}$ School of Mathematics and Statistics, Henan University of Science and Technology, Luoyang, \\ China \\ aliuchunf@haust.edu.cn, 'Ipllpl_Ipl@163.com, 'libaoan@haust.edu.cn \\ * The corresponding author
}

Keywords: University teaching quality; Guarantee system; Analytic hierarchy model; Fuzzy comprehensive evaluation model; Fuzzy neural network model

\begin{abstract}
In this paper, the modeling analysis about teaching quality guarantee system is discussed. In Henan University of Science and Technology, for example, first the corresponding questionnaire survey has been made, then using the method of analytic hierarchy structure and fuzzy comprehensive evaluation to establish the teaching quality guarantee system of the secondary fuzzy comprehensive evaluation model, finally the fuzzy neural network model of university teaching quality guarantee system is established.
\end{abstract}

\section{高校教学质量保障体系的模糊综合评判和神经网 络模型实证分析}

刘春峰 $^{1, a}$, 李培峦 ${ }^{2, b}$, 李保安 ${ }^{2, c}$

1. 河南科技大学 教务处，中国 河南 洛阳 471023

2. 河南科技大学 数学与统计学院，中国 河南 洛阳 471023

aliuchunf@haust.edu.cn, ${ }^{b}$ lpllpl_lpl@163.com, ${ }^{c}$ libaoan@ @aust.edu.cn

摘要: 探讨了关于高校教学质量保障体系的建模分析. 以河南科技大学为例, 首先进行了问卷 调查，然后利用层次分析结构法和模糊综合评判建立高校教学质量保障体系的二级模糊综合 评判模型, 最后建立了高校教学质量保障体系的模糊神经网络模型。

关键词：高校教学质量；保障体系；层次分析结构模型；模糊综合评判模型；模糊神经网络 模型

\section{1. 引言}

《国家中长期教育改革和发展规划纲要 2010-2020 年》指出在新的历史时期, 提高人才培养 质量是高等教育发展的核心任务。要提高人才培养质量, 就要完善高校的教学质量保障体系。 教学质量保障体系是指全面提高教学质量的校内工作体系和运行机制。保障体系有五个部分, 即教学管理与组织系统、教学信息收集与处理系统、教学评价系统、教学条件保障系统和教 学质量信息的反馈系统。随着我国高等教育大众化进程的加快, 构建全面、科学、高效的内 部教学质量保障体系成为高校需要关注和解决好的重要问题. 目前对于高校的教学质量保障 
体系的研究很多, 很多学者是从教育学和管理学的角度进行研究 $[1,2,3]$, 也有还有学者是以 经济学中的观点来探讨高校教学质量 [4], 本文从统计学的角度来研究高校的教学质量保障体 系。

\section{2. 调查问卷设计与分析}

充分考虑到高校教育质量保障体系的评估是一个系统工程, 影响其的因素众多, 在设计问卷 过程中，遵守问卷设计的原则：有明确的主题；结构合理、逻辑性强; 通俗易懂和便于资料 的校验、整理和统计。

主要将河南科技大学作为调查地点, 发放 360 份问卷, 共获得 345 份有效调查问卷, 回收率 达到 95.8\%。其中大一的有 95 份, 大二的有 97 份, 大三和大四各有 65 份和 88 份。被调查 者的专业分布如下: 理科 131 人，工科 71 人，文科 62 人，农医 81 人，调查范围是比较广， 也比较均匀。从回收的调查问卷中也可以看出问卷完成情况良好, 并具有一定的代表性, 基 本保证了信息来源的真实性和调查的有效性。表 1 是关于问卷调查所有项目结果的统计表。 从表 11 可分析得知, 在调查项目中教学资源、师资水平和教师评价的评价获得良好和优秀的 百分比都大于 $80 \%$, 这表明学生对这些评价项目是充分肯定的, 但是还是有改进之处。特别 是在职称结构这个评价项目中, 较差的百分比达到 3.32\%, 更说明了问题的所在, 教师的职 称结构还有待完善。从上表中可以分析得知, 教学管理和信息网建设的被评为优秀和良好的 比率接近 90\%, 获得了被调查者的肯定, 但是也存在一定的缺陷, 教师信息和毕业生信息的 缺乏，使得这两个评价项目获得较差评价的百分比大于 $4 \%$ 。 
表 1 各评价项目结果分布统计表

\begin{tabular}{|c|c|c|c|c|c|c|c|c|}
\hline 项目 & 评价内容 & & 合计 & 优秀 & 良好 & 一般 & 及格 & 较差 \\
\hline \multirow{6}{*}{$\begin{array}{l}\text { 教 } \\
\text { 学 } \\
\text { 资 } \\
\text { 源 }\end{array}$} & \multirow{2}{*}{ 教学设备 } & 调查人数 & 345 & 173 & 120 & 37 & 8 & 7 \\
\hline & & 比例 (\%) & 100 & 50.23 & 34.99 & 10.71 & 1.96 & 2.11 \\
\hline & \multirow{2}{*}{ 实验设备 } & 调查人数 & 345 & 182 & 121 & 30 & 8 & 4 \\
\hline & & 比例（\%） & 100 & 52.79 & 35.14 & 8.6 & 2.41 & 1.06 \\
\hline & \multirow{2}{*}{ 图书资源 } & 调查人数 & 345 & 207 & 74 & 39 & 20 & 5 \\
\hline & & 比例（\%） & 100 & 60.03 & 21.57 & 11.01 & 5.73 & 1.66 \\
\hline \multirow{6}{*}{$\begin{array}{l}\text { 师 } \\
\text { 资 } \\
\text { 水 } \\
\text { 平 }\end{array}$} & \multirow{2}{*}{ 学历结构 } & 调查人数 & 345 & 164 & 139 & 27 & 10 & 5 \\
\hline & & 比例（\%） & 100 & 47.51 & 40.19 & 7.79 & 2.96 & 1.55 \\
\hline & \multirow{2}{*}{ 职称结构 } & 调查人数 & 345 & 137 & 152 & 6 & 38 & 2 \\
\hline & & 比例（\%） & 100 & 39.67 & 44.04 & 1.81 & 11.16 & 3.32 \\
\hline & \multirow{2}{*}{ 高职称教师上课率 } & 调查人数 & 345 & 146 & 152 & 36 & 9 & 2 \\
\hline & & 比例（\%) & 100 & 42.23 & 44.19 & 10.41 & 2.56 & 0.61 \\
\hline \multirow{6}{*}{$\begin{array}{l}\text { 教 } \\
\text { 师 } \\
\text { 评 } \\
\text { 价 }\end{array}$} & \multirow{2}{*}{ 课程建设 } & 调查人数 & 345 & 210 & 104 & 5 & 21 & 5 \\
\hline & & 比例（\%） & 100 & 60.79 & 30.09 & 1.32 & 6.23 & 1.57 \\
\hline & \multirow{2}{*}{ 教学方法 } & 调查人数 & 345 & 160 & 149 & 23 & 10 & 3 \\
\hline & & 比例（\%） & 100 & 46.51 & 43. 19 & 6.73 & 2.86 & 0.71 \\
\hline & \multirow{2}{*}{ 教学效果 } & 调查人数 & 345 & 200 & 92 & 37 & 6 & 10 \\
\hline & & 比例（\%） & 100 & 57.92 & 26.54 & 10.71 & 1.81 & 3.02 \\
\hline \multirow{4}{*}{$\begin{array}{l}\text { 教 } \\
\text { 学 } \\
\text { 理 } \\
\text { 管 }\end{array}$} & \multirow{2}{*}{ 管理规章 } & 调查人数 & 345 & 226 & 90 & 23 & 5 & 1 \\
\hline & & 比例（\%） & 100 & 65.46 & 26.09 & 6.79 & 1.36 & 0.3 \\
\hline & \multirow{2}{*}{ 考试管理 } & 调查人数 & 345 & 160 & 146 & 21 & 11 & 7 \\
\hline & & 比例（\%） & 100 & 46.49 & 42.23 & 6.14 & 3.12 & 2.02 \\
\hline \multirow{6}{*}{$\begin{array}{l}\text { 信 } \\
\text { 息 } \\
\text { 建 } \\
\text { 设 }\end{array}$} & \multirow{2}{*}{ 学生信息网建设 } & 调查人数 & 345 & 240 & 71 & 18 & 10 & 6 \\
\hline & & 比例 (\%) & 100 & 69.43 & 20.45 & 5.13 & 3.03 & 1.96 \\
\hline & \multirow{2}{*}{ 教师信息网建设 } & 调查人数 & 345 & 196 & 107 & 5 & 10 & 27 \\
\hline & & 比例 (\%) & 100 & 56.82 & 31.08 & 1.32 & 2.83 & 7.95 \\
\hline & \multirow{2}{*}{ 毕业生信息网建设 } & 调查人数 & 345 & 133 & 155 & 8 & 35 & 14 \\
\hline & & 比例（\%） & 100 & 38.63 & 45.04 & 1.91 & 10.16 & 4.26 \\
\hline
\end{tabular}

\section{3. 教育质量保障体系的二级模糊综合评判模型}

3.1. 层次分析结构模型与指标权重的确定

3.1 .1 给出一级指标和二级指标

选择教学质量高校保障体系中 5 个一级指标和 14 个二级指标, 其中 5 个一级指标为: $A 1$ 教 学资源、 $A 2$ 师资水平、 $A 3$ 教学评价、 $A 4$ 教学管理和 $A 5$ 教学信息网建设。14 个二级指标为: $A 1$ 教学资源包括 $B 1$ 教学设备、 $B 2$ 实验设备、 $B 3$ 图书资源; $A 2$ 师资水平包括 $B 4$ 学历结构、 $B 5$ 职称结构、 $B 6$ 高职称教师上课率; $A 3$ 教学评价包括 $B 7$ 教材建设、 $B 8$ 教学方法、 $B 9$ 教 学效果; $A 4$ 教学管理包括 $B 10$ 管理规章、 $B 11$ 考试管理; $A 5$ 教学信息网建设包括 $B 12$ 学生信 息网建设、B13教师信息网建设、B14 毕业生信息网建设。

2.1.2 构造成对比较矩阵

在教学质量高校保障体系中一级指标对比见下表。 
表 2 一级指标对比表

\begin{tabular}{llllll}
\hline$Z$ & $A 1$ & $A 2$ & $A 3$ & $A 3$ & $A 3$ \\
\hline$A 1$ & 1 & 2 & $1 / 2$ & $1 / 5$ & $1 / 2$ \\
$A 2$ & $1 / 2$ & 1 & $1 / 2$ & $1 / 2$ & $1 / 5$ \\
$A 3$ & 2 & 2 & 1 & $1 / 2$ & $1 / 3$ \\
$A 4$ & 5 & 2 & 2 & 1 & 2 \\
$A 5$ & 2 & 5 & 3 & $1 / 2$ & 1 \\
\hline
\end{tabular}

过 MATLAB 计算可得 $\lambda_{\text {max }}=5.3620$, 特征向量 $\eta=\left(\begin{array}{c}-0.2089 \\ -0.1663 \\ -0.2978 \\ -0.7151 \\ -0.5732\end{array}\right)$, 查表可知 $R I=1.12$, 计算可得 $C I=0.0905, C R=\frac{C I}{R I}=0.0808<0.1$ ，通过一致性检验。

将特征向量归一化可得一级指标权向量 $\eta^{\prime}=\left(\begin{array}{l}0.1065 \\ 0.0848 \\ 0.1518 \\ 0.3646 \\ 0.2923\end{array}\right)$

二级指标包括教学设备、学历结构、教材建设、管理规章和学生信息网建设等 14 个二级指标。 以下是教学资源的二级指标对比表。

表 3 二级指标对比表

\begin{tabular}{cccc}
\hline $\boldsymbol{Z}$ & $\boldsymbol{B} 1$ & $\boldsymbol{B} 2$ & $\boldsymbol{B} 3$ \\
\hline $\boldsymbol{B} 1$ & 1 & 2 & $1 / 2$ \\
$B 2$ & $1 / 2$ & 1 & $1 / 2$ \\
$B 3$ & 2 & 2 & 1 \\
\hline
\end{tabular}

通过 MATLAB 计算可得 $\lambda_{\text {max }}=3.0536$, 特征向量 $\eta=\left(\begin{array}{l}0.5053 \\ 0.3183 \\ 0.8021\end{array}\right)$, 查表可知 $R I=0.58$, 计算可得 $C I=0.0268, C R=\frac{C I}{R I}=0.046<0.1$, 通过一致性检验。将特征向量归一化可得二级指标权向 量 $\eta^{\prime}=\left(\begin{array}{l}0.3108 \\ 0.1958 \\ 0.4934\end{array}\right)$ 。

以下是师资水平的二级指标对比表。 


\begin{tabular}{cccc}
\hline$Z$ & $B 4$ & $B 5$ & $B 6$ \\
\hline$B 4$ & 1 & $1 / 5$ & $1 / 2$ \\
$B 5$ & 5 & 1 & 3 \\
$B 6$ & 2 & $1 / 3$ & 1 \\
\hline
\end{tabular}

通过 MATLAB 计算可得 $\lambda_{\text {max }}=3.0037$, 特征向量 $\eta=\left(\begin{array}{c}-0.1747 \\ -0.9281 \\ -0.3288\end{array}\right)$, 查表可知 $R I=0.58$, 计算可得 $C I=0.0019, C R=\frac{C I}{R I}=0.0033<0.1$, 通过一致性检验。将特征向量归一 化可得二级指标权向量 $\eta^{\prime}=\left(\begin{array}{l}0.1220 \\ 0.6483 \\ 0.2297\end{array}\right)$ 。

以下是教学评价的二级指标对比表。

表 5 二级指标对比表

\begin{tabular}{cccc}
\hline $\boldsymbol{Z}$ & $\boldsymbol{B 7}$ & $\boldsymbol{B} 8$ & $\boldsymbol{B} 9$ \\
\hline $\boldsymbol{B} 7$ & 1 & $1 / 5$ & $1 / 2$ \\
$B 8$ & 5 & 1 & 3 \\
$B 9$ & 2 & $1 / 3$ & 1 \\
\hline
\end{tabular}

通过 MATLAB 计算可得 $\lambda_{\text {max }}=3.0536$, 特征向量 $\eta=\left(\begin{array}{c}0.5053 \\ 0.3183 \\ 0.8021\end{array}\right)$, 查表可知 $R I=0.58$, 计算可得 $C I=0.0268, C R=\frac{C I}{R I}=0.0462<0.1$, 通过一致性检验。将特征向量归一化可得二级指标权向 量 $\eta^{\prime}=\left(\begin{array}{l}0.3108 \\ 0.1958 \\ 0.4934\end{array}\right)$ 。教学管理的二级指标数为 2 , 通过一致性检验, 权向量 $\eta^{\prime}=\left(\begin{array}{l}0.6667 \\ 0.3333\end{array}\right)$ 。 以下是教学信息网建设的二级指标对比表。

表 6 二级指标对比表

\begin{tabular}{cccc}
\hline$Z$ & $B 12$ & $B 13$ & $B 14$ \\
\hline$B 12$ & 1 & $1 / 5$ & $1 / 2$ \\
$B 13$ & 5 & 1 & 3 \\
$B 14$ & 2 & $1 / 3$ & 1 \\
\hline
\end{tabular}


通过 MATLAB 计算可得 $\lambda_{\text {max }}=3.0055$, 特征向量 $\eta=\left(\begin{array}{c}-0.8902 \\ -0.4132 \\ -0.1918\end{array}\right)$, 查表可知

$R I=0.58$, 计算可得 $C I=0.0268, C R=\frac{C I}{R I}=0.0462<0.1$, 通过一致性检验。将特征向量归一 化可得二级指标权向量 $\eta^{\prime}=\left(\begin{array}{l}0.5954 \\ 0.2764 \\ 0.1283\end{array}\right)$ 。

3.2. 二级模糊综合评判模型的构建

$A 1$ 评估隶属度计算:

评估矩阵为:

$R_{21}=\left(\begin{array}{ccccc}50.23 & 34.99 & 10.71 & 1.96 & 2.11 \\ 52.79 & 35.14 & 8.6 & 2.41 & 1.06 \\ 60.03 & 21.57 & 11.01 & 5.73 & 1.66\end{array}\right)$

权向量为 $W_{21}=(0.3108,0.1958,0.4934)$, 对 $A 1$ 的评估等级的隶属度为:

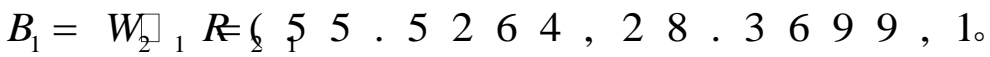

按照同样的方法及过程还可以分别计算出 $A 2, A 3, A 4$ 和 $A 5$ 隶属度。

$B_{2}=(41.2145,43.6048,4.5150,8.1842,2.4816)$

$B_{3}=(56.5779,30.9034,7.0123,3.3893,2.1170)$

$B_{4}=(59.1373,31.4695,6.5734,1.9466,0.8733)$

$B_{5}=(57.6811,30.6431,6.0265,3.4713,2.1724)$

由上可知，我校高校教育质量保障体系的评估识别矩阵为:

$R=\left(\begin{array}{ccccc}55.5264 & 28.3699 & 10.4363 & 3.9067 & 1.6807 \\ 41.2145 & 43.6048 & 4.5150 & 8.1842 & 2.4816 \\ 56.5779 & 30.9034 & 7.0123 & 3.3893 & 2.1170 \\ 59.1373 & 31.4695 & 6.5734 & 1.9466 & 0.8733 \\ 61.9999 & 26.5451 & 3.6643 & 3.8898 & 3.9109\end{array}\right)$

各一级指标的权重 $W=(0.1065,0.0848,0.1518,0.3646,0.2923)$, 则我校的综合评估向量为: $B=W \square R=(57.6811,30.6431,6.0265,3.4713,2.1724)$ 。将 评估等级进行赋值 $Z=\left\{\mathrm{z}_{1}, \mathrm{z}_{2}, \mathrm{z}_{3}, \mathrm{z}_{4}, \mathrm{z}_{5}\right\}=(100,80,60,40,20)$, 则我校的综合评估等级得分为

$C=B \square Z^{T}=(57.6811,30.6431,6.0265,3.4713,2.1724)\left(\begin{array}{c}100 \\ 80 \\ 60 \\ 40 \\ 20\end{array}\right) * 1 \%=87.6345$,

所以该校的教育保障体系的评估等级为良好。 


\section{4. 教育质量保障体系的模糊神经网络模型}

4.1. 模糊神经网络模型的原理

模糊神经网络 (Fuzzy neural network, 简称 FNN) 是用神经网络表示出模糊规则和隶属函数, 将生成的神经网络用于实现模糊推理, 再利用误差反传算法训练神经网络, 从而提高系统精 度、修改隶属函数、求模糊规则。这个过程首先是由输入内容的模糊化过程; 然后由模糊推 理过程实行逻辑推理; 最终进行决策判断。它的各个节点的连接权值和其他参数可以通过学 习不断调整。

模糊神经网络的评判模型适用于高校教育质量保障体系的评估。模糊评判过程具体为:
(1) 寻找因素集 $U=\left\{\mathrm{u}_{1}, \mathrm{u}_{2}, \ldots, \mathrm{u}_{n}\right\}$;
(2) 建立评判集 $V=\left\{v_{1}, v_{2}, \ldots v_{m}\right\}$;

(3) 找出评判矩阵 $R=\left(\mathrm{r}_{i j}\right)_{n \times m}$;

（4）建立权重集 $A=\left(\mathrm{a}_{1}, \mathrm{a}_{2}, \ldots, \mathrm{a}_{n}\right)$;

（5）设评判矩阵 $R$ 每个单因素到评语集的最大隶属度集 $G=\left(\mathrm{g}_{1}, \mathrm{~g}_{2}, \ldots, \mathrm{g}_{n}\right)^{T}$ ，其中 $g_{i}=r_{i 1} \vee r_{i 2} \vee \ldots \vee r_{i m}, i=1,2, \ldots, n$ 。则 $B(j)=\sum_{i=1}^{n} a_{i}\left(g_{i}-r_{i j}\right), j=1,2, \ldots m$ 。

对于指标 $A$ 来说, 如果因素 $u_{i}$ 越接近 $g_{i}$, 则 $u_{i}$ 就越接近 $g_{i}$ 所对应的评语等级。所以我们用 $u_{i}$ 偏离 $g_{i}$ 的程度来衡量其优劣。 $B(\mathrm{j})$ 越小, 评判结果就越接近 $u_{i}$ 对应的评判等级, 评判结果就 是 $B$ 中最小的数对应的等级。

确定权重的算法：设 $b_{j}^{*}, j=1,2 \ldots, m$ 为期望输出值。给定初始值 $w_{i}(0)$, 学习样本 $R^{1}, R^{2}, \ldots, R^{P}$ 。 期望输出向量 $B_{1}^{*}, B_{2}^{*}, \ldots, \mathrm{B}_{P}^{*}$, 误差 $\varepsilon\left(\varepsilon=\frac{1}{2} \sum_{j=1}^{m}\left(\mathrm{~b}_{j}^{*}-b_{j}\right)^{2}\right)$ 。记 $w_{i}(\mathrm{t})$ 为 $t$ 时刻第 $i$ 个输入的权重, $i=1,2, \ldots, n$ 。

(1) 随机选出一组样本 $R^{P 1}$ 和期望输出 $B_{P 1}^{*}$, 记 $P_{1}=1$ 。计算实际网络输出 $B_{P 1}=\left(\mathrm{b}_{1}^{P 1}, \mathrm{~b}_{2}^{P 1}, \ldots, \mathrm{b}_{m}^{P 1}\right)$;

(2) 计算 $\square B_{P 1}-B_{P 1}^{*} \square=\sqrt{\sum_{i=1}^{m}\left(\mathrm{~b}_{i}^{P 1}-\mathrm{b}_{i}^{* P 1}\right)^{2}} \quad$ ，若 $\square B_{P 1}-B_{P 1}^{*} \square \mathcal{E}$, 转下一步，否则转回第 2 步;

（3）调整权值 $\Delta w_{i}=w_{i}(\mathrm{t}+1)-\mathrm{w}_{i}(\mathrm{t})=\sum_{j} \frac{\partial E}{\partial b_{j}} \frac{\partial b_{j}}{\partial w_{i}}=\sum_{j}\left(\mathrm{~b}_{j}^{*}-\mathrm{b}_{j}\right) r_{i j}$ ；

(4) 按照新的权值计算输出和总误差。若都满足 $\square B_{P 1}-B_{P 1}^{*} \llbracket<\varepsilon$, 则停止, 否则转回第 2 步。 最终算法:

(1) 给定初始值 $R^{(0)}$, 期望输出向量 $B^{*}=\left(\mathrm{b}_{1}^{*}, \mathrm{~b}_{2}^{*}, \ldots, \mathrm{b}_{m}^{*}\right)$, 并给定误差 $\varepsilon$, 记 $R^{t}$ 为 $t$ 时刻的评判 矩阵。

(2) 计算每个指标的最大承属度 $G^{(\mathrm{t})}=\left(\mathrm{g}_{1}^{t}, \mathrm{~g}_{2}^{t}, \ldots, \mathrm{g}_{n}^{t}\right)^{T}$, 其中

$g_{i}^{(\mathrm{t})}=g_{i 1}^{(\mathrm{t})} \vee g_{i 2}^{(\mathrm{t})} \vee \ldots \vee g_{i m}^{(\mathrm{t})}, \quad i=1,2, \ldots, n$;

(3) 计算判断矩阵的偏差矩阵

$$
R^{d(\mathrm{t})}=\left[\begin{array}{cccc}
g_{1}^{(\mathrm{t})}-r_{11}^{(\mathrm{t})} & g_{1}^{(\mathrm{t})}-r_{12}^{(\mathrm{t})} & \ldots & g_{1}^{(\mathrm{t})}-r_{1 m}^{(\mathrm{t})} \\
g_{2}^{(\mathrm{t})}-r_{21}^{(\mathrm{t})} & g_{2}^{(\mathrm{t})}-r_{21}^{(\mathrm{t})} & \ldots & g_{2}^{(\mathrm{t})}-r_{2 m}^{(\mathrm{t})} \\
\ldots & \ldots & \ldots & \ldots \\
g_{n}^{(\mathrm{t})}-r_{n 1}^{(\mathrm{t})} & g_{n}^{(\mathrm{t})}-r_{n 2}^{(\mathrm{t})} & \ldots & g_{n}^{(\mathrm{t})}-r_{n m}^{(\mathrm{t})}
\end{array}\right]=\left[\begin{array}{cccc}
r_{11}^{d(\mathrm{t})} & r_{12}^{d(\mathrm{t})} & \ldots & r_{1 m}^{d(\mathrm{t})} \\
r_{21}^{d(\mathrm{t})} & r_{22}^{d(\mathrm{t})} & \ldots & r_{2 m}^{d(\mathrm{t})} \\
\ldots & \ldots & \ldots & \ldots \\
r_{n 1}^{d(\mathrm{t})} & r_{n 2}^{d(\mathrm{t})} & \ldots & r_{n m}^{d(\mathrm{t})}
\end{array}\right] 。
$$

(4) 计算权重向量 $W^{t}=\left(w_{1}^{(\mathrm{t})}, w_{2}^{(\mathrm{t})}, \ldots, w_{n}^{(\mathrm{t})}\right)$, 其中 $w_{i}^{(\mathrm{t})}=\sum_{j=1}^{m} r_{i j}^{d(\mathrm{t})} / \sum_{i=1}^{n} \sum_{j=1}^{m} r_{i j}^{d(\mathrm{t})}$;

(5) 给定初始值 $R^{d(\mathrm{t})}$, 计算出 $B^{(\mathrm{t})}=W \square R^{\mathrm{d}(\mathrm{t})}=\left(\mathrm{b}_{1}^{(t)}, \mathrm{b}_{2}^{(t)}, \ldots, \mathrm{b}_{m}^{(t)}\right)$; 
(6) 计算 $\square B^{(\mathrm{t})}-B^{*} \square=\sqrt{\sum_{i=1}^{m}\left(\mathrm{~b}_{i}^{(\mathrm{t})}-\mathrm{b}_{i}^{*}\right)^{2}}$ ，若 $\square B^{(\mathrm{t})}-B^{*} \square>\varepsilon$ 。转下一步，否则转第 2 步;

(7) 记 $E=\frac{1}{2} \sum_{J=1}^{m}\left(\mathrm{~b}_{j}^{*}-\mathrm{b}_{j}^{(\mathrm{t})}\right)^{2}$ 为修正评判矩阵。因为

$\Delta r_{i j}=r_{i j}^{t+1}-\mathrm{r}_{i j}^{(\mathrm{t})}=\sum_{j} \frac{\partial E}{\partial b_{j}} \frac{\partial b_{j}}{\partial r_{i j}}=\sum_{j}\left(\mathrm{~b}_{j}^{*}-\mathrm{b}_{j}^{(\mathrm{t})}\right) w_{i}^{(\mathrm{t})}$,

则 $r_{i j}(\mathrm{t}+1)=\mathrm{r}_{i j}(\mathrm{t}) \vee\left[\underset{j}{\vee}\left(\left(\mathrm{b}_{j}^{(\mathrm{t})}-\mathrm{b}_{j}^{*}\right) \wedge w_{i}\right)\right]$;

(8) 求出 $R^{(t+1)}$, 重复第二步和第五步, 如满足 $\square B^{(t+1)}-B^{*} \square<\varepsilon$, 转下一步, 否则回到第 2 步;

(9) 给定 $R^{(\mathrm{t})}$ 与 $R^{(\mathrm{t}+1)}$ ，对误差 $\varepsilon, \max \left\{\left(\mathrm{r}_{i j}{ }^{(\mathrm{t}+1)}-\mathrm{r}_{i j}{ }^{t}\right)\right\}<\varepsilon$, 则 $R^{(\mathrm{t}+1)}$ 可以，迭代停止，否则回到第 2 步。

用以上算法求解神经网络, 修改隶属函数, 求出模糊规则, 提高了推理系统的求解的精度。

4.2. 模糊神经网络的计算结果

基于调查所得数据, 经过上述计算可得如下结果。

表 7 一级指标等级计算结果

\begin{tabular}{cccccc}
\hline 一级指标 & 优秀 & 良好 & 一般 & 及格 & 较差 \\
\hline 教学资源 & 54.5264 & 29.3699 & 10.5346 & 3.8068 & 1.7623 \\
师资水平 & 43.2034 & 44.6708 & 3.5045 & 8.2843 & 0.337 \\
教师评价 & 56.5079 & 30.9064 & 7.2013 & 3.2896 & 2.0948 \\
教学管理 & 57.1213 & 32.4689 & 7.5764 & 1.0762 & 1.7572 \\
$\begin{array}{c}\text { 信息网建 } \\
\text { 设 }\end{array}$ & 60.1232 & 28.5453 & 3.7634 & 3.6798 & 3.8883 \\
\hline
\end{tabular}

从输出结果可以看出一级指标的等级计算结果与模糊综合评判模型的判断矩阵是非常相似 的, 这也验证了模糊神经网络模型的精确性。从输出结果得出的一级指标的偏导数结果, 如 表 8 。

表 8 偏导数计算结果

\begin{tabular}{cccccc}
\hline 一级指标 & 教学资源 & 师资水平 & 教师评价 & 教学管理 & 信息网建设 \\
\hline 偏导数 & 0.9985 & 1.1783 & 1.4274 & 2.2521 & 2.0213 \\
\hline
\end{tabular}

由上表可知, 对高校教育质量保障体系影响较大的依次是教学管理、信息网建设、教师评价、 师资水平和教学资源, 与上文计算所得各指标的权重大小相一致, 这也验证了模糊神经网络 模型的精确性。因此本文的模型及结果比相关文献 $[5,6,7,8,9]$ 要精确, 具有一定的理论意义 和实际应用价值。

\section{5. 致谢}

基金项目: 国家自然科学基金项目(11101126，11261010), 河南省高等教育教学改革研究项 目(2014SJG LX195)，河南科技大学重大教育教学改革研究项目(2014ZD-007)。 


\section{参考文献}

[1] 鲁晓. 高校教学质量保障体系的构建 [J]. 现代教育管理, 2009 (4)：88-90.

[2] 龙金智. 关于高校教学质量保障体系的建立和完善[J]. 考试周刊, 2009 （31）：197-198.

[3] 漆玲玲. 高校教育质量模糊综合评估的探讨 $[\mathrm{J}]$. 中国电力教育，2014（27）：13-15.

[4] 徐向艺. 高等学校教学质量管理的范畴、原则与体系 [J]. 高等理科教育, 2004(1): 40-45.

[5] 丁家玲, 叶金华. 层次分析法和模糊综合评判在教师课堂教学质量评价中的应用 $[\mathrm{J}]$. 武 汉大学学报（社会科学版），2003，56（2）：241-245.

[6] 任春玉, 王晓博, 王万学. 基于模糊综合评价的高校教学质量保障体系研究 [J]. 黑龙江 教育（高教研究与评估）, 2006（7-8）：182-183.

[7] 刘云辉, 李钟慎. 改进型模糊神经网络模型的构造 [J]. 华侨大学学报（自然科学版）, 2010, 31 (3) : 256-259.

[8] 赵春元. 基于层次分析的教学质量模糊综合评价模型及应用 $[\mathrm{J}]$. 沈阳工程学院学报 (自 然科学版)，2011, 7 (2) : 185-189.

[9] 曾松伟, 吴胜达, 胡海根, 周素茵. 基于层次分析法的高校教学质量评价改革 [J]. 绍兴 文理学院学报，2012，32（10）：89-94.

[10]姜启源, 谢金星, 叶俊. 数学模型 $[\mathrm{M}]$. 第四版. 北京：高等教育出版社, 2011年.

[11]Jiang Qiyuan, Xie Jinxing, Ye Jun. Mathematical Model. China Higher Education Press, 2011, p. 249-269.

\section{Acknowledgement}

This research was financially supported by the national natural science foundation of China (11101126, 11261010), the Research Project of Higher Education Reform in Henan (2014SJG LX195) and the Teaching Reform Research Planning Project of Henan University of Science and Technology (2014ZD-007)

\section{References}

[1] Lu Xiao. The Construction of College Teaching Quality Guarantee System. Modern Education Management. 2009, No. 4, p. 88-90.

[2] Long Jinzhi. About the Establishment and improvement of the university teaching quality guarantee system. Examination weekly. 2009, No. 31, p. 197-198.

[3] Qi Lingling. The Discussion about University Education Quality base on Fuzzy Comprehensive Evaluation. China Electric Power Education. 2014, No. 27, p. 13-15.

[4] Xu Xiangyi. Category, Principle and System of Uinversity teaching quality management. Higher Education of Science. 2004, No. 1, p. 40-45.

[5] Ding Jialing, Ye Jinhua. On AHP Model and Fuzzy Judgement in Evaluation of Undergraduate Teaching Quality. Journal of Wuhan University of Hydraulic and Electrical Engineering (Social Sciences Edition). Vol. 56 (2003) No. 2, p. 241-245.

[6] Ren Chunyu, Wang Xiaobo, Wang Wanxue. The Research of University Teaching Quality Security System based on the Fuzzy Comprehensive Evaluation. Heilongjiang Education(Higher Education Research \& Appraisal). 2006, No. 7-8, p. 182-183. 
[7] Liu Yunhui, Li Zhongshen. Construction of Improved Fuzzy Neural Network Model. Journal of Huaqiao University (Natural Science). Vol. 31 (2010) No. 3, p. 256-259.

[8] Zhao Chunyuan. Application of fuzzy judgment based on AHP model in the evaluation of teaching quality. Journal of Shenyang Institute of Engineering (Natural Science). Vol. 7 (2011) No. 2, p. 185-189.

[9] Zeng Songwei, Wu Shengda, Hu Haigen, et al. Reform of University Teachers'Performance Based on Analytic Hierarchy Process. Journal of Shaoxing University. Vol. 32 (2012) No. 10, p. 89-94.

[10]Jiang Qiyuan, Xie Jinxing, Ye Jun. Mathematical Model. China Higher Education Press, 2011, p. 249-269.

作者简介: 刘春峰 (1972-), 男, 河南洛阳, 副教授, 主要研究方向为高等教育学、数学 建模, E-mail: 1iuchunf@haust. edu.cn。 